\title{
Erratum to: Noncommutativity as a Colimit
}

\author{
Benno van den Berg • Chris Heunen
}

Published online: 5 December 2012

(C) Springer Science+Business Media Dordrecht 2012

\section{Erratum to: Appl Categor Struct DOI 10.1007/s10485-011-9246-3}

The main purpose of this erratum is to correct a claim we made in our paper "Noncommutativity as a colimit" (Applied categorical structures, Volume 20, Number 4, August 2012, pp. 393-414) at the bottom of page 411, in between Proposition 9 and Lemma 4. Fortunately, our mistake does not invalidate any of the results in the paper or make them less relevant.

Let us first define the categories CstaredTopos and LocaledTopos.

Definition 1 The category CstaredTopos has as objects pairs $(T, A)$ of a topos $T$ and an internal $\mathrm{C}^{*}$-algebra $A$. A morphism $(T, A) \rightarrow\left(T^{\prime}, A^{\prime}\right)$ consists of a geometric morphism $F: T^{\prime} \rightarrow T$ and an internal *-morphism $\varphi: A^{\prime} \rightarrow F^{*}(A)$ in $T^{\prime}$.

The category LocaledTopos has as objects pairs $(T, L)$ of a topos $T$ and an internal locale object $L$. A morphism $(T, L) \rightarrow\left(T^{\prime}, L^{\prime}\right)$ consists of a geometric morphism $F: T^{\prime} \rightarrow T$ and an internal locale morphism $\varphi: F^{*}(L) \rightarrow L^{\prime}$ in $T^{\prime}$.

The online version of the original article can be found at http://dx.doi.org/10.1007/s10485-011-9246-3.

B. van den Berg was supported by the Netherlands Organisation for Scientific Research (NWO).

C. Heunen was supported by the Netherlands Organisation for Scientific Research (NWO). Part of this work was performed while the author visited the IQI at Caltech.

B. van den Berg

Universiteit Utrecht, Mathematisch Instituut, PO Box 80010,

3508 TA, Utrecht, the Netherlands

e-mail: B.vandenBerg1@uu.nl

C. Heunen $(\varangle)$

Department of Computer Science, University of Oxford, Wolfson Building, Parks Rd,

OX1 3QD, Oxford, United Kingdom

e-mail: heunen@cs.ox.ac.uk 
By Proposition 9, Bohrification determines a functor PCStar ${ }^{\text {op }} \rightarrow$ CstaredTopos. $^{\circ}$ It is not immediately clear that composing this functor with taking internal spectra results in a functor PCStar ${ }^{\text {op }} \rightarrow$ LocaledTopos: that would require that taking the internal Gelfand spectrum of a commutative $\mathrm{C}^{*}$-algebra commutes with inverse image functors of geometric morphisms. Contrary to what we state in the paper, these two constructions do indeed commute. As was pointed out to us by Steven Vickers, the description of the internal spectrum as a Grothendieck site is geometric (as is clear from [3]) and therefore preserved by inverse image functors. So Bohrification does yield a functor PCStar ${ }^{\text {op }} \rightarrow$ LocaledTopos after all.

Nevertheless, there is no obvious functor LocaledTopos $\rightarrow$ Loc and therefore we still do not have a "spectrum functor" PCStar" $\rightarrow$ Loc. For that, it still seems necessary to restrict to a special class of morphisms in PCStar (like those that reflect commeasurability).

Additionally, let us also note that the clause $f(a+i b)=f(a)+i f(b)$ in Definition 4 should be dropped: this condition only makes sense when $a \odot b$, in which case it is superfluous. Consequently, the proof of Theorem 5 can be considerably shortened by putting $m(a)=f_{A\langle a\rangle}(a)$, making it completely analogous to the proof of Theorem 1 . 